Lin et at., Afr J Tradit Complement Altern Med. (2010) 7(4):307-314

\title{
EFFECT OF ACHYRANTHES BIDENTATA POLYSACCHARIDES ON THE EXPRESSION OF BCL-2 AND BAX IN HEPATIC TISSUES AFTER EXHAUSTIVE EXERCISE IN RATS
}

\section{Jinyang Lin $^{1 *}$, Zhuoying Zhang ${ }^{2}$ and Ying Shan ${ }^{3}$}

${ }^{1}$ Department of Physical Education, Central South University, Changsha City, Hunan Province, 410083, China. ${ }^{2}$ Department of Physical Education, Hunan Normal University, Changsha City, Hunan Province, 410081, China. ${ }^{3}$ Department of Physical Education and Military Training, Zhejiang University of Technology, Hangzhou City, Zhejiang Province, 310014, China.

*E-mail: jinyewlin@yahoo.com.cn, JyLhnzz@126.com

\begin{abstract}
This study aims to assess the effects of Achyranthes bidentata polysaccharides (ABPS) on the expression of bcl-2 and bax in hepatic tissues after exhaustive exercise in order to provide theoretical support for the application of ABPS in the field of sports nutrition. Thirty male Sprague-Dawley rats were randomized into three groups, each consisting of 10 rats: Normal control group (NCG), Exhausting exercises control group (EECG), ABPS treated group (ATG). ABPS were fed orally by gastric intubation to rats of ABPS treated group (ATG) once daily for 7 days. Control animals (EECG and NCG) received the same amount of isotonic sodium chloride solution. Exhaustive exercise was performed on a rodent treadmill. The SP (streptavidin peroxidase) method for immunohistochemical staining was adopted to test the protein expression of bax and bcl-2 in the hepatic tissues of the rats. Exhausting exercises increased bax protein expression of hepatic tissues of rats and bax/bcl-2 ratio dramatically, but a decreased bcl-2 protein expression. In the rats fed ABPS orally by gastric intubation, the bax protein expression and bax/bcl-2 ratio obviously decreased, while bcl-2 protein expression increased. The result indicated that bax and bcl-2 co-regulated the exercise-induced hepatocyte apoptosis. Feeding ABPS orally by gastric intubation to rats can inhibit the hepatocyte apoptosis in exhaustive exercise.
\end{abstract}

Key words: achyranthes bidentata polysaccharides, apoptosis, bcl-2, bax, exhaustive exercise

\section{Introduction}

Achyranthes bidentata (Amaranthaceae, Chinese Phonetic Name-Niuxi) is an erect, annual herb distributed in hilly districts of China, Korea, Japan and India. The plant has been proven to have wide application in traditional and folk medicines in these, as well as, other eastern countries (Nikolov et al., 1996). The whole plant, and particularly the roots, have been shown to contain various saponins, sterols, polysaccharides and alkaloids (Bishit and H.Sandhu, 1990; Marcone et al., 2003; Shi et al., 2006) and has well-defined expectorant, anti-inflammatory, antipyretic, antirheumatic and diuretic activities. The bioactive polysaccharides isolated from its root is called Achyranthes bidentata polysaccharides (ABPS). ABPS have been reported to possess immunopotentiating, antisenile, antiradiation, antitumor and hypoglycemic effects (Xiang and $\mathrm{Li}$, 1993; Tan and Deng, 2002; Shao et al., 2002; Li, 2004).

Apoptosis is a highly regulated form of cell death that is characterized by specific morphological, biochemical, and molecular events (Steller, 1995; Jia, 2007). It is essential for the normal development of multicellular organisms and is involved in cell turnover in healthy adult tissues (Duke et al., 1996; Carraro and Franceschi, 1997; Siu et al., 2004). Apoptosis also plays a critical role in removing unwanted and potentially dangerous cells, such as tumor cells and cells infected by viruses (Williams, 1991; Vaux et al., 1994). Disorders such as cancer, AIDS, Alzheimer's disease, and rheumatoid 
Lin et at., Afr J Tradit Complement Altern Med. (2010) 7(4):307-314

arthritis are thought to be, at least partially, a result of aberrant regulation of apoptosis (Duke et al., 1996).

Recently, apoptosis has gained the interest of many exercise scientists because, in addition to necrotic cell death, evidence indicates that apoptotic cell death also occurs with exercise. There is evidence for certain relationship between apoptotic and exercises. For example, glucocorticoids, growth-factor withdrawal, reactive oxygen species (ROS), a rise in intracellular $\mathrm{Ca} 2+$ levels, and tumor necrosis factor (TNF) are some of the signals that can induce apoptosis (Phaneuf and Leeuwenburgh, 2001). Some of these factors originate from the extracellular milieu (glucocorticoids, TNF) and will interact with intracellular or extracellular proteins that may trigger cell death. Increases in glucocorticoid secretion, intracellular calcium concentrations, and reactive oxygen species production have been shown to occur during strenuous exercise and have the potential to induce apoptosis (Phaneuf and Leeuwenburgh, 2001; Mooren et al., 2004; Wang and Huang, 2005).

Apoptotic signaling induces apoptosis primarily through three types of complex pathways. They include 1) cytokine/Fas receptor-driven pathway, 2) mitochondrial-driven pathway, and 3) endoplasmic reticulum/ $/ \mathrm{Ca}^{2+}$-driven pathway (Hengartner, 2000; Kwak et al., 2006). Among them, mitochondrial-mediated pathway, including the bcl-2 family is the best characterized and believed to be critical in regulating apoptosis (Centurione et al., 2003; Liu et al., 1998; Ruetten et al., 2001; Hengartner, 2000). The mitochondrial bcl-2 pathway includes a family of competing, membrane-bound 1) antiapoptotic "gatekeepers," including bcl-2 and bcl-xl, and 2) proapoptotic "gatecrashers," including bax, bad, and bid (Hengartner, 2000). The ratio of proapoptotic to antiapoptotic proteins (e.g., bax/bcl-2) regulates myonuclei integrity and cell survival by controlling mitochondrial membrane permeability (Hengartner, 2000). This work was designed to shed light on effects of Achyranthes bidentata polysaccharides (ABPS) on the expression of bcl-2 and bax in hepatic tissues after exhaustive exercise in order to provide theoretical support for the application of ABPS in the field of sports nutrition.

\section{Materials and Methods}

Plant materials

The roots of Achyranthes bidentata (voucher No. CST09-484)were bought from the market of traditional Chinese medicinal materials in Changsha, China and identified ccording to the identification standard of the eighth edition of Pharmacopeia of People's Republic of China (2005 PPRC)..

\section{Preparation of crude polysaccharides}

The roots of Achyranthes bidentata were moistened with water and refluxed with $80 \%$ ethanol for $1 \mathrm{hr}$ twice to remove impurity. The volume of ethanol used every time was five times that of the plant material. The residue was dried and then extracted with boiling water. The filtrate was filtered and condensed under ordinary pressure. The concentrated solution was deproteined with $3 \%$ trichloroacetic acid three times. The supematant was concentrated again, and its $\mathrm{pH}$ value was adjusted to 10 with $10 \% \mathrm{NaOH}$. Pre-cooled $95 \%$ ethanol was added into the concentrated supematant till the final concentration of ethanol was $80 \%$ and kept 4 to $5 \mathrm{hrs}$ until the precipitate settled and the precipitate was dissolved in water, then the polysaccharides was precipitated again with ethanol at the concentration of $80 \%$. The precipitate was then dehydrated with $90 \%, 95 \%, 100 \%$ ethanol in that order. The crude polysaccharides was dried at $80^{\circ} \mathrm{C}$ in vacuum (Tan and Deng, 2002; Hou and Wang, 2008).

\section{Determination of polysaccharide content}

The contents of polysaccharides were measured by the phenolsulphuric acid method using glucose as standard (Cuesta et al., 2003). The basic protocol was followed, with the modifications indicated below. The sugar solution $(2 \mathrm{~mL})$ and 
Lin et at., Afr J Tradit Complement Altern Med. (2010) 7(4):307-314

the phenol solution $(2 \mathrm{~mL})$ were added to each screw cap tube $(18 \times 180 \mathrm{~mm})$, which was capped and vortex-stirred. Then 10 $\mathrm{mL}$ of concentrated sulfuric acid was added slowly down along the side of the tube. The tubes were then closed, vortex-stirred for 5 seconds and incubated for $2 \mathrm{~min}$ at $100^{\circ} \mathrm{C}$ water bath. All tubes were allowed to cool down to room temperature before measuring the absorbance at $490 \mathrm{~nm}$ using distilled water as blank in the Multiskan Spectrum Microplate Spectrophotometer (Thermo Labsystem) (Zhou et al., 2007; Xu and Gu, 2009). Standard curve line was drawn, the regression equation was derived on the basis of data processing $\mathrm{C}=15.4624 \mathrm{~A}-0.1035(\mathrm{R}=0.9993)$. The contents of polysaccharides were finally determined to be $5.68 \mathrm{mg} / \mathrm{g}$.

\section{Animals and diet}

Male Sprague-Dawley rats 7 to 9 weeks old were obtained from Hunan biological supplier (Changsha, China) and were bred in the animal facilities at Central South University. Rats were housed in a regulated temperature $\left(23 \pm 2{ }^{\circ} \mathrm{C}\right)$ and humidity (55\%) controlled room with a 12:12-h light-dark cycle. They were provided a standard pelleted diet (Zhengda Ltd, Changsha, China) and water ad libitum. The ingredient and nutrient composition of the control basal diet fed to rats is given in Table 1.

Table 1: Ingredient and nutrient composition of the control basal diet fed to rats

\begin{tabular}{cc||cc}
\hline Ingredient & Amount $(\mathrm{g} / \mathrm{kg})$ & Ingredient & Amount $(\mathrm{g} / \mathrm{kg})$ \\
\hline Casein & 21 & Cellulose & 50 \\
Corn starch & 440 & Soybean oil & 50 \\
Sucrose & 100 & Vitamin mix & 10 \\
Maltose dextrin & 100 & Mineral mix & 35 \\
\hline
\end{tabular}

Note: Other ingredients include choline bitartrate $(2 \mathrm{~g} / \mathrm{kg})$ and t-butyl hydroquinone $(0.008 \mathrm{~g} / \mathrm{kg})$. Proximate analysis: $21 \%$ crude protein, $5 \%$ crude fat, $4 \%$ crude fiber, $8 \%$ ash.

Prior permission for animal use and approval of the protocol were obtained from the Institutional animal ethics committee. Thirty rats were divided into the following 3 groups, each consisting of 10 animals.

Group 1 : Normal control group (NCG) fed with isotonic sodium chloride solution

Group 2: Exhausting exercises control group (EECG) fed with isotonic sodium chloride solution

Group 3: ABPS treated group (ATG) fed with ABPS (100mg/kg. d)

ABPS were fed orally by gastric intubation to rats of ABPS treated group once daily for 7 days. Control animals (EECG and NCG) received the same amount of isotonic sodium chloride solution. Animals of Group 1 were not exercised; Animals of Group 2 and Group 3 were exercised.

\section{Experimental protocol}

Exhaustive exercise was performed on a rodent treadmill with the following protocols. In the adaptive period, rats were accustomed to treadmill running for 7 days. Then, the rats of exhaustive exercise group were subjected to graded treadmill running starting at $10 \%$ grade, $15 \mathrm{~m} / \mathrm{min}$ for $15 \mathrm{~min}$ followed by a gradual increase in the treadmill speed and time to $25 \mathrm{~m} / \mathrm{min}$ for $15 \mathrm{~min}, 30 \mathrm{~m} / \mathrm{min}$ for $30 \mathrm{~min}, 35 \mathrm{~m} / \mathrm{min}$ for $60 \mathrm{~min}, 40 \mathrm{~m} / \mathrm{min}$ for $30 \mathrm{~min}, 45 \mathrm{~m} / \mathrm{min}$ for $30 \mathrm{~min}$ until exhaustion (Figure 1) (Hsieh et al., 2006; Niu et al., 2008). 
Lin et at., Afr J Tradit Complement Altern Med. (2010) 7(4):307-314

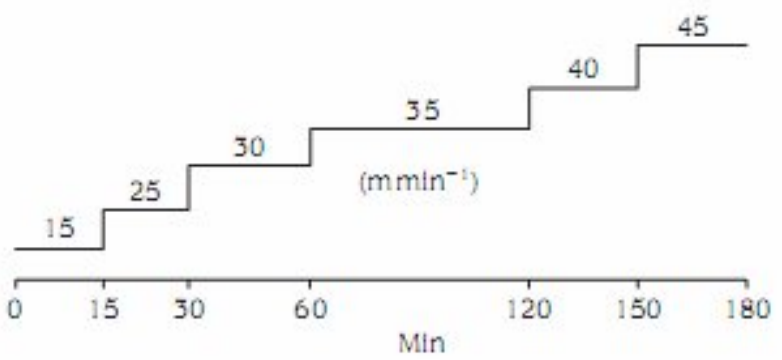

Figure 1: Exhaustive exercise protocol

Electrical shocks were used sparingly in exhaustive exercise groups to motivate the animals to run. Exhaustion was defined as the inability of the rats to run on the treadmills, despite electrical shock (Hsieh et al., 2006).

\section{Tissue preparation}

At the end of exhaustive exercise, the rats were anesthetized with ether (an anesthesia chamber was utilized as the induction method of delivering volatile anesthetic agent to the rats); ether was volatilized by placing it on cotton balls at the bottom of the jar. The rats after anesthetization were killed by decapitation. Rats of normal control group were also killed at the same time as the exhaustive exercise group. The hepatic tissues were extracted from rats, and the obtained tissues were treated with $4 \%$ paraformaldehyde in $0.1 \mathrm{M}, \mathrm{pH} 7.4$ phosphate buffered saline (PBS) for 24 hrs before the embedding in paraffin. The tissues were cut into serial sections of 6 um thickness (Chae et al., 2002).

\section{Bax and bcl-2 protein expression}

The SP (streptavidin peroxidase) method for immunohistochemical staining was adopted to test the protein expression of bax and bcl-2 in the hepatic tissues of the rats. The process was carried out as existing literature: the tissues were cut into slices of 4um, and dewaxing hydration was conducted. The first antibody used was the rabbit anti-mouse anti-bax and bcl-2 polyclonal antibody. The second antibody was labeled by dropping horseradish peroxidase. This was used as a negative control by replacing the first antibody with phosphate buffered saline (PBS). The slices were then coloured with 3,3'-diaminobenzidine (DAB) and then with hematoxylin stain. Finally, the slices were fixed with resin (Zhong et al., 2003; Ma et al., 2009). After bax and bcl-2 protein expression of hepatic tissues was observed under light microscope, the comprehensive judgment was carried out based on the percentage of positive cells. The presence of brown punctate or granular substances in the cells is an indication of positive staining.

\section{Statistical analysis}

Values were presented as mean and standard deviation for nomal distribution variables or median and quartile range for highly skewed variables. The significance of differences among the three groups was tested using the Kruskal-Wallis test for highly skewed data and analysis of variance (ANOVA) for normal distribution data. Mutiple comparisons were subjected to Bonfferoni correction test. The Chisquare test was used to evalute equality of frequencies for discrete variables. Correlations were tested using the Spearman rank correlation coefficients. $p<0.05$ was considered statistical significant and all statistical analyses were conducted using SPSS version 11.5 for windows. 
Lin et at., Afr J Tradit Complement Altern Med. (2010) 7(4):307-314

\section{Results}

\section{Change in bax protein expression in hepatic tissues}

In the hepatic tissues of rats, the protein expression of bax was investigated using the SP method. Compared exhausting exercises control group (EECG) and ABPS treated group (ATG) with normal control group (NCG), the protein expression of bax had significant statistic difference $(p<0.05)$, and compared ABPS treated group (ATG) with exhausting exercises control group (EECG), the protein expression of bax had also significant statistic difference (Table 2, $p<0.05$ ).

Table 2: The comparison of bax among three groups $(n=10)$

\begin{tabular}{ll}
\hline Group & Bax \\
\hline Normal control group (NCG) & $12.69 \pm 3.94$ \\
Exhausting exercises control group (EECG) & $22.17 \pm 5.38 *$ \\
ABPS treated group (ATG) & $17.49 \pm 4.83 * \Delta$ \\
\hline
\end{tabular}

Note: ${ }^{*} p<0.05$, as compared with Normal control group; ${ }^{\triangle} p<0.05$, as compared with Exhausting exercises control.

\section{Change in bcl-2 protein expression in hepatic tissues}

In the hepatic tissues of rats, the protein expression of bcl-2 was investigated using the SP method. Compared exhausting exercises control group (EECG) with normal control group (NCG), the protein expression of bcl-2 had significant statistic difference $(p<0.05)$. Compared normal control group (NCG) and ABPS treated group (ATG) with exhausting exercises control group (EECG), the protein expression of bcl-2 had also significant statistic difference (Table 3, $p<0.05$ ).

Table 3: The comparison of bcl-2 among three groups $(\mathrm{n}=10)$

\begin{tabular}{ll}
\hline Group & Bcl-2 \\
\hline Normal control group (NCG) & $19.16 \pm 3.87^{\triangle}$ \\
Exhausting exercises control group (EECG) & $13.86 \pm 4.53^{\star}$ \\
ABPS treated group (ATG) & $17.31 \pm 4.89^{\triangle}$ \\
\hline
\end{tabular}

Note: ${ }^{*} p<0.05$, as compared with Normal control group; ${ }^{\triangle} p<0.05$, as compared with Exhausting exercises control.

\section{Change in bax/bcl -2 ratio}

Comparing exhausting exercises control group (EECG) and ABPS treated group (ATG) with normal control group (NCG), the bax/bcl -2 ratio had significant statistic difference $(p<0.05)$. Comparing normal control group (NCG) and ABPS treated group (ATG) with exhausting exercises control group (EECG), the bax/bcl -2 ratio had also significant difference (Table 4, $p<0.05$ ).

Table 4: The comparison of bax/bcl -2 ratio among three groups $(n=10)$

\begin{tabular}{ll}
\hline Group & Bax/bcl-2 \\
\hline Normal control group (NCG) & $0.66^{\triangle}$ \\
Exhausting exercises control group (EECG) & $1.60^{\star}$ \\
ABPS treated group (ATG) & $1.01^{\star} \triangle$ \\
\hline
\end{tabular}


Lin et at., Afr J Tradit Complement Altern Med. (2010) 7(4):307-314

Note: ${ }^{*} p<0.05$, as compared with Normal control group; ${ }^{\triangleright} p<0.05$, as compared with Exhausting exercises control.

\section{Discussion}

The results of the change in bax protein expression in hepatic tissues indicated that exhaustive exercises could lead to increase in bax protein expression in hepatic tissues of rats dramatically. However, after the rats were fed ABPS orally by gastric intubation the bax protein expression in hepatic tissues of rats decreased under the same circumstances.

The results of the change in bcl-2 protein expression in hepatic tissues indicated that exhaustive exercises could lead to decrease in bcl-2 protein expression in hepatic tissues of rats. But, after the rats were fed ABPS orally by gastric intubation, the bcl-2 protein expression in hepatic tissues of rats increased under the same circumstances. As the ratio of Bax/Bcl-2 in hepatic tissues of rats and hepatocyte apoptosis rate are positively correlated (Kim et al., 2005), the results indicated that feeding ABPS orally by gastric intubation to rats can inhibit the hepatocyte apoptosis in exhaustive exercise.

Apoptosis is orchestrated by the sequential activation of caspases, a family of cysteine proteases with specificity for aspartic acid residues. Two pathways may mediate apoptosis: 1) the death receptors pathway (tumour necrosis factor, Fas ligand or TRAIL receptors) that activates caspase 8 and 10;2) the mitochondrial pathway that activates caspase 9. Both pathways lead to effector caspases (3,6 and 7) (Igney and Krammer, 2002). Bcl-2 is antiapoptotic for the mitochondria pathway, whereas Bax is a proapoptotic factor activated by p53 (Zhu et al., 2006).

$\mathrm{Bcl}-2$ is an integral, membrane-associated protein with antiapoptotic and perhaps antioxidative effects. It may also regulate intracellular concentrations of $\mathrm{Ca}^{2+}$ ions. Bax is another member of the family of bcl-2-related proteins. It has an extensive aminoacid homology with bcl-2. Both proteins homodimerise and heterodimerise with each other. Whether the cell will live or die may depend on the level of either protein; while bcl-2 prevents death, bax is a death promoter (Lichnovsky et al., 2000). The ratio of bax/bcl-2 determines the trend of apoptosis (Lee et al., 2005).

Apoptotic cell death induced by exercise in tissues exposed to specific stresses (calcium, glucocorticoids, radicals) may be a normal process used to remove partially damaged cells. Excessive and/or eccentric exercise may cause significant mechanical damage, followed by an inflammatory response, leading to necrosis and apoptosis. Apoptosis is a partially reversible process and therefore, therapeutic interventions could be tested to attenuate this process (Phaneuf and Leeuwenburgh, 2001).

In the present study, we found that exhausting exercises could make bax protein expression in hepatic tissues of rats and bax/bcl-2 ratio dramatically increases, and bcl-2 protein expression obviously decreases. The results indicated that bax and bcl-2 co-regulated the exercise-induced hepatocyte apoptosis. Bax promotes the apoptosis as up-regulated genes, while bcl-2 inhibits apoptosis as down-regulated genes, and this may be the gene regulation mechanism of exercise-induced hepatocyte apoptosis in rat liver. While after being fed ABPS orally by gastric intubation to rats, the bax protein expression and bax/bcl-2 ratio obviously decreased, bcl-2 protein expression obviously increased. The results indicated that feeding ABPS orally by gastric intubation to rats can inhibit the hepatocyte apoptosis in exhaustive exercise.

\section{Conclusions}

Bax and bcl-2 co-regulated the exercise-induced hepatocyte apoptosis. Bax promotes the apoptosis as up-regulated genes, while bcl-2 inhibits apoptosis as down-regulated genes. Feeding ABPS orally by gastric intubation to rats can inhibit the hepatocyte apoptosis in exhaustive exercise. The results provide theoretical support for the application of ABPS in the field of sports nutrition. 
Lin et at., Afr J Tradit Complement Altern Med. (2010) 7(4):307-314

\section{References}

1. Bishit, G. and H, Sandhu. (1990). Chemical constituents and antimicrobial activity of Achyranthes bidentata. Journal of the Indian Chemical Society, 67: 1002--1003.

2. Carraro, U. and Franceschi, C. (1997). Apoptosis of skeletal and cardiac muscles and physical exercise. Aging (Milano), 9: 19-34.

3. Centurione, L., Di Giulio, C., Cacchio, M., Rapino, M., Bosco, D., Grifone, G., Sabatini, N., Bianchi, G., Antonucci, A. and Cataldi, A. (2003). Correlations between protein kinase c (zeta) signaling and morphological modifications during rat heart development and aging. Mechanisms of Ageing and Development, 124: 957-966.

4. Chae, H.S., Bach, J.H., Yoo, S.J. and Kim, D.J. (2002). The Expression of Bcl-2 and Bax in Rat Hepatic Erythropoiesis. Korean J. Anatomy, 35: 143-152.

5. Cuesta, G., Suarez, N., Bessio, M.I., Ferreira, F. and Massaldi, H. (2003). Quantitative determination of pneumococcal capsular polysaccharide serotype 14 using a modification of phenol-sulfuric acid method. J. Microbiological Methods, 52: 69-73.

6.Duke, R.C., Ojcius, D.M. and Young, J.D. (1996). Cell suicide in health and disease. Scientific American, 275: $80-87$.

7. Hengartner, M.O. (2000). The biochemistry of apoptosis. Nature, 407: 770-776.

8. Hou, J.P. and Wang, C.X. (2008). Study on the Extracting and Purifying Process of Achyranthes Bidentata Polysaccharides. China Pharmacy, 19: 2822-2824.

9. Hsieh, C.C., Lee, M.Y., Chen, C. C., Hsu, J. J., Lu, H.K. and Wang, C.J. (2006). Hibiscus protocatechuic acid supplementation reduces oxidative stress induced by exhaustive exercise in rat muscle. Journal of Exercise Science and Fitness, 4: 59-63.

10. Igney FH. and Krammer PH (2002). Death and anti-death: tumor resistance to apoptosis. Nat Rev Cancer, 2: $277-288$

11. Jia, L. (2007). Sports stress and the apoptosis of the cells. Journal of Lanzhou University (Medical Sciences), 33: $72-76$.

12. Kim WH, Lee JW, Suh YH, Hong SH, Choi JS, Lim JH, Song JH, Gao B, Jung MH (2005). Exposure to Chronic High Glucose Induces \{beta\}-Cell Apoptosis Through Decreased Interaction of Glucokinase With Mitochondria: Downregulation of Glucokinase in Pancreatic \{beta\}-Cells. Diabetes. 54: 2602-2611.

13. Kwak, H.B., Song, W. and Lawler, J.M. (2006). Exercise training attenuates age-induced elevation in Bax/Bcl-2 ratio, apoptosis, and remodeling in the rat heart. The FASEB Journal, 36: 1476-1483.

14. Lee, S.G., Yum, J.S. and Moon, H.M. (2005). Analysis of mannose-binding lectin 2 (MBL2) genotype and the serum protein levelsin the Korean population. Molecular Immunology. 42: 969-970.

15. Li, H.Q. (2004). Research of achyranthes bidentata polysaccharides on hypoglycemic effect in experimental diabetic mice. Anhui Medical and Pharmaceutical Journal, 8: 326-327.

16. Lichnovsky, V., Procházková, J., Erdösová, B., Nepoîitková, D. and Âernochová, D. (2000). Apoptosis and expression of bcl-2 and bax during early human embryogenesis. Scripta Medica, 73: 245-250.

17. Liu, L., Azhar, G., Gao, W., Zhang, X. and Wei, J.Y. (1998). Bcl-2 and Bax expression in adult rat hearts after coronary occlusion: age-associated differences. American J. Physiol., 275: R315-R322.

18. Ma, H.B.; Lin, H.Y.; Feng, H. and Putheti, R. (2009). Effects of angelica polysaccharide on hepatocytes apoptosis induced by exhaustive exercise. African J. Microbiology Research, 3: 774-777.

19. Marcone, M.F., Aliee, H. and Kakuda, Y. (2003). Chemical characterization of Achyranthes bidentata seed. Food 
Lin et at., Afr J Tradit Complement Altern Med. (2010) 7(4):307-314

Chemistry, 81: 7-12.

20. Mooren, F.C., Lechtermann, A. and Völker, K. (2004). Exercise-induced apoptosis of lymphocytes depends on training status. Medicine and Science in Sports and Exercise, 36: 1476-1483.

21. Nikolov, S., Thuan, N. and Zheljazkov, V. (1996). Flavonoids from Achyranthes Bidentata BC. Acta Horticulturae, 426: $75-78$.

22. Niu, A.J., Wu, J.M., Yu, D.H. and Wang, R. (2008). Protective effect of Lycium barbarum polysaccharides on oxidative damage in skeletal muscle of exhaustive exercise rats. International J. Biological Macromolecules, 42: 59-63.

23. Phaneuf, S. and Leeuwenburgh, C. (2001). Apoptosis and exercise. Medicine \& Science in Sports \& Exercise, 33: 393-396.

24. Ruetten, H., Badroff, C., Ihling, C., Zeiher, A. M. and Dimmeler, S. (2001). Inhibition of caspase-3 improves contractile recovery of stunned myocardium, independent of apoptosis-inhibitory effects. J. American College of Cardiology, 38: 2063-2070.

25. Shao, S.J., Mai, L. and Chen, Y. (2002). The effect of achyranthes bidentata polysaccharide on mice red blood cell immunological function. Chinese Remedies \& Clinics, 2: 281-282.

26. Shi, C.J., Zhou, Y.D., Zhang, J.B. and Tian, G.Y. (2006). Researches of polysaccharides from Achyrnthes bidentata. Chinese Journal of New Drug, 15: 1330-1334.

27. Siu, P.M., Bryner, R.W., Martyn, J. K. and Always, S. E. (2004). Apoptotic adaptations from exercise training in skeletal and cardiac muscles. The FASEB Journal, 18: 1150-1154.

28. Steller, H. (1995). Mechanisms and genes of cellular suicide. Science, 267: 1445-1449.

29. Tan, F. and Deng, J. (2002). Analysis of the Constituents and Antisenile Function of Achyranthes bidentata Polysaccharides. Acta Phytotaxonomica Sinica, 44: 795-798.

30. Vaux, D.L., Haecker, G. and Strasser, A. (1994). An evolutionary perspective on apoptosis. Cell, 76: 777-779.

31. Wang, J.S. and Huang, Y. H. (2005). Effects of exercise intensity on lymphocyte apoptosis induced by oxidative stress in men. European Journal of Applied Physiology, 95: 290-297.

32. Williams, G.T. (1991). Programmed cell death: apoptosis and oncogenesis. Cell, 65: 1097-1098.

33. Xiang, D.B. and Li, X.Y. (1993). Effects of Achyranthes bidentata polysaccharides on interleukin-1 and tumor necrosis factor-alpha production from mouse peritoneal macrophages. Zhongguo Yao Li Xue Bao, 14: 332-336.

34. Xu, H.D. and Gu, X.M. (2009). Extraction and Determination of the Polysaccharides in Achyranthes bidentta. Science Technology and Engineering, 9: 107-109.

35. Zhong, X.; Yi J. and He, X.B. (2003). Hepatocyte apoptosis and the expression of Bcl-2 and Bax in Yin-jaundice rats. J. Chinese Integrative Medicine, 1: 116-118.

36. Zhou, X.W., Lin, J. and Tang, K.X. (2007). Cytotoxic activities of Coriolus versicolor (Yunzhi) extracts on human liver cancer and breast cancer cell line. African J. Biotechnology, 6: 1740-1743.

37. Zhu, C.Q., Shih, W. and Ling, C.H. (2006). Immunohistochemical markers of prognosis in non-small cell lung cancer: a review and proposal for a multiphase approach to marker evaluation. J Clin Pathol , 59: 790-800. 